\title{
PREHISTORIA DE LA SEGURIDAD ALIMENTARIA, GENERALIDADES Y PERIFERIAS: COSTA RICA 1950-1970
}

Prehistory of Food Security, generalities and peripheries: Costa Rica 1950-1970

\author{
José David Ramírez Roldán \\ Universidad de Costa Rica, Costa Rica \\ jdr_r@hotmail.com \\ Álvaro Sanabria Chacón \\ Universidad de Costa Rica, Costa Rica \\ asanabriac@gmail.com \\ Daniel Díaz Pérez \\ Universidad de Costa Rica, Costa Rica \\ daiaz81@gmail.com
}

Recibido: 29-01-2019

Aprobado: 29-03-2019

José David Ramírez Roldán es Bachiller en Historia por la Universidad de Costa Rica, investigador independiente, profesor y columnista. Ha colaborado en investigación en la Casa Presidencial de Costa Rica y el Centro de Investigación en Identidad y Cultura Latinoamericanas (CIICLA). $\mathrm{Ha}$ publicado diversos artículos sobre neografía, educación e historia colonial y contemporánea centroamericana en los siglos XVIII$\mathrm{XX}$.

Álvaro Sanabria Chacón es Licenciado en Medicina y Cirugía por la Universidad de Costa Rica, además es estudiante de Historia en la misma institución

superior.

\section{RESUMEN}

El presente artículo estudia la evolución de la seguridad alimentaria en Costa Rica como parte de una experiencia nacional e internacional que tiene origen desde los años cincuenta y se consolido conceptualmente en la década de los setenta. En otras palabras, en este trabajo se analiza a profundidad las políticas públicas y multinacionales que fomentaron las acciones de la seguridad alimentaria y nutricional en el país.

Palabras clave: seguridad alimentaria; Costa Rica; política de la salud; política de desarrollo; nutrición

\section{ABSTRACT}

This paper studies the evolution of food security in Costa Rica as part of a national and international experience that began in the 1950 s and became conceptually consolidated in the 1970s. In other words, this work analyzes in depth the public and multinational policies that promoted the actions of food and nutrition security in the country.

Keywords: food security; Costa Rica; nutrition policy; development policy; nutricional 
Se ha desempeñado en el deporte, la medicina geriátrica, la fotografía y el rescate de la cultura cartaginesa en Paraíso desde la historia local.

Daniel Díaz Pérez es estudiante de último año en la carrera de Historia de la Universidad de Costa Rica. Es investigador independiente de tema relacionados con la salud, el consumo y la producción de alimentos.

\section{Introducción}

La evolución en la concepción de la seguridad alimentaria y otros términos afines (ejemplo: seguridad nutricional) tienen una existencia de apenas 40 años, considerando sus constantes cambios según la normatividad oficial del momento (Clay, 2002). Dicho concepto tiene su origen a mediados de los años 70, cuando la Cumbre Mundial sobre la Alimentación llegó a definirla como un plan para el suministro de alimentos, desde su disponibilidad y estabilidad nacional e internacional para controlar los precios de los alimentos básicos de consumo diario.

En la actualidad casi la totalidad de los países del mundo tienen como meta asegurar a su población los recursos nutricionales necesarios para la vida por medio de los alimentos de consumo común. Lamentablemente, no todos han logrado un progreso considerable, para el caso de Costa Rica sí hay un desarrollo privilegiado en la historia de la salud pública, no solo en comparación con el istmo centroamericano, sino que también a nivel mundial.

El fenómeno de la seguridad alimentaria, basándose en las definiciones que se rige $\mathrm{El}$ Programa Mundial de Alimentos (WFP en sus siglas en inglés), está conformado por cuatro factores: la disponibilidad, el acceso, la estabilidad y la utilización (FAO-FIDAPMA, 2014, p. 13). La disponibilidad se refiere a la cantidad, calidad y diversidad que una población dada goza para su manutención. El acceso está vinculado con la capacidad de infraestructura, ingreso económico y posibilidad física, por ejemplo, si un país tiene adecuadas carreteras o medios de transporte, el ingreso promedio da abasto para comprar alimentos. El factor de estabilidad está relacionado con qué tan dependiente es un país de su producción o de las importaciones para abastecer a la mayoría de la población, además de eso, también puede estar relacionado con la volatilidad de los precios, y la inestabilidad política. Por último, el factor de la utilización se divide en dos variables, primero, el uso que le da la población a los alimentos que posee, un ejemplo de ellos es el saneamiento, o el acceso de agua potable; $y$ el segundo, es sobre los efectos de la mala nutrición (FAO-FIDA-PMA, 2014, p. 13).

Bajo estas series de definiciones, se puede concebir la idea de seguridad alimentaria como una condición que engloba a los individuos, su capacidad de conseguir alimentos y el consumo que estos les den. Este estado de salud, no sólo se le puede ver como un indicio de bienestar, ya que, además depende de qué tan efectivo sea en un lugar 
determinado, por lo que podría mostrar la situación de socioeconómica en un lugar dado; y de ahí qué tan eficaces son los programas de acción social.

Por consiguiente, el problema o cuestionamiento en esta investigación recae en preguntarse: ¿cómo se desarrollaron en Costa Rica durante los años cincuenta, sesenta y setenta las políticas públicas y multinacionales que fomentaron la seguridad alimentaria y nutricional?

Asimismo, como hipótesis sostenemos que, posiblemente con la experiencia de la tradición de la intervención social de los años treinta y cuarenta en Costa Rica, como parte de la continuidad de esas políticas desde los años cuarenta, se llegó a ofrecer un crecimiento y una mayor facilidad a los organismos que tenían tareas para el mejoramiento de la producción alimentaria y la nutrición de centros o periferias; y con ello las estrategias predominantemente internacionales evidenciaron un marco nacional previo como referencia para la vía exitosa del desarrollo social que se venía dando. De alguna manera esto le ofreció al país cierto papel destacado a nivel del istmo centroamericano.

De hecho, el interés de este trabajo se presenta en poder definir esa experiencia histórica de preocupación sobre los alimentos consumidos, a partir de la determinación de sus condiciones de producción y los campos de intervención, de forma general, por parte del Estado a nivel nacional; y de forma específica, entorno a las periferias del país. También, buscamos presentar los objetivos de la gestión seguridad alimentaria, con el fin de identificar los principales organismos internacionales e instituciones nacionales implicados en la gestión de la seguridad alimentaria que participaron en los sistemas de Seguridad Alimentaria y Nutricional desde los años cincuenta y sesenta en Costa Rica. Además, se busca identificar la génesis del derecho alimentario, o sea, la Ley General de Salud aplicada en 1973 .

\section{Antecedentes de la seguridad alimentaria}

La obligación de proveer a la población de los alimentos necesarios para satisfacer las necesidades básicas, ha sido un mandato que las autoridades gubernamentales han tenido presente a través de la historia. La relación en cuanto a oferta y demanda de productos básicos de consumo, constituye un elemento que permite establecer un estatus de bienestar y estabilidad en la población. No obstante, la conciencia de esto, los instrumentos de medición y las estrategias de acción no siempre tuvieron la atención requerida y se dejó a la libre la regulación propia del mercado interno. 
En 1948, en la Declaración Universal de los Derechos Humanos, se reconoce la alimentación como un derecho humano. El artículo 25 de esta declaración establece que: "Toda persona tiene derecho a un nivel de vida adecuado que le asegure, así como a su familia, la salud y el bienestar, y en especial la alimentación" (Ministerio de Salud, 2011, p. 11). Esto obliga a los gobiernos suscritos a esta declaración proporcionar las debidas políticas para asegurar un abastecimiento digno de los alimentos básicos, además del resto de condiciones de bienestar y salud. En dicho pacto se establece la garantía del derecho a la alimentación, el cual se considera cumplido cuando las personas tienen acceso físico y económico, en todo momento, a la alimentación adecuada y a los medios para obtenerla. En el caso nuestro, el artículo 7 de la Constitución Política da rango constitucional a los tratados internacionales: "Los tratados públicos, los convenios internacionales y los concertados, debidamente aprobados por la Asamblea Legislativa tendrán desde su promulgación o desde el día que ellos designen autoridad superior a las leyes".

Todos estos antecedentes gestaron en la segunda mitad del siglo XX, nuevos acuerdos internacionales se centraron en la necesidad de asegurar la alimentación para los países. Así, en 1966 se suscribe el Pacto Internacional de derechos económicos, sociales y culturales; en 1996 se celebra la Cumbre Mundial de la Alimentación, y se planteó el derecho a una adecuada alimentación, su efectiva aplicación señala que existe seguridad alimentaria cuando toda la población, y en todo momento, tiene acceso físico, social y económico a alimentos seguros y nutritivos que satisfacen sus necesidades dietéticas y preferencias alimentarias para una vida activa y saludable (Ministerio de Salud, 2011, p. 11). En el 2002 se incluyen en la Cumbre Mundial sobre la Alimentación directrices en apoyo al derecho a la alimentación adecuada en el contexto de la seguridad alimentaria. Los Objetivos de Desarrollo del Milenio promulgados por las Naciones Unidas y/o las metas y objetivos de la Organización Mundial para la Agricultura y la Alimentación, sitúan la política de seguridad alimentaria y nutricional como un instrumento para el combate a la pobreza en los sectores más vulnerables, la generación de ingresos, la productividad agrícola y el acceso de bienes para consumo nacional (Ministerio de Salud, 2011, p. 12).

Sin embargo, como mencionábamos en un inicio, los gobiernos siempre se han preocupado por atender la demanda de alimentación. En el caso nuestro, es posible registrar estas intenciones en diversos momentos de la historia, de manera especial cuando prima una coyuntura internacional que afecta directa o indirectamente las economías dependientes e impacta la distribución interna del mercado.

Por definición, la seguridad alimentaria y nutricional se entiende como: el "Estado en el cual todas las personas gozan, en forma oportuna y permanente, de acceso físico, económico y social, a los alimentos que necesitan, en calidad y cantidad, para su adecuado consumo y utilización biológica, garantizándoles un estado de bienestar general que coadyuve al logro de su desarrollo" (Ministerio de Salud, 2011, p. 12). Esta conceptualización aparece en la segunda mitad del siglo XX y es acogida en los diferentes foros internacionales que abogan por el derecho humano a una nutrición sana y efectiva. 
No obstante, los planteamientos y los esfuerzos por suministrar a la población de estas condiciones se pueden rastrear desde algunas décadas antes.

\section{La crisis de una guerra mundial}

El estallido de la Segunda Guerra Mundial colocó nuevamente al mercado internacional contra la pared, el intercambio comercial entre las naciones europeas y los países proveedores se redujo a insumos para enfrentar la guerra. Para el caso de economías como la costarricense, que era completamente dependiente del mercado mundial, tanto para la venta de sus productos como también para la importación de otros, el conflicto conllevó a una escasez de productos de primera necesidad que no se producían en el país. Esto provocó que se implementaran políticas de gobierno para palear la crisis. El 29 de agosto de 1942, vía decreto se crea la Secretaría de Agricultura y Ganadería, su primer Secretario fue Mariano R. Montealegre, el cual en su rendición de cuentas frente

al Congreso en 1943 manifestaba las condiciones difíciles que enfrentaba el sector y los grandes retos que se avecinaban:

\footnotetext{
Creada, como lo fue, en medio de la guerra feroz que conmueve al Mundo y con el objetivo primordial de aumentar la producción en decadencia, no era posible esperar que durante mi gestión se llevaran a cabo labores excepcionales ya que no se contaba ni con tiempo ni con elementos, ni con dinero. (Ministerio de Agricultura e Industria, 1943, p. v).
}

Efectivamente, las condiciones que imperaban al inicio de la década de los cuarenta, recrudecidas por los azotes indirectos de la guerra, ponen de manifiesto una situación de apremio para el gobierno y sus instituciones. Si bien se crea esta Secretaría con un objetivo muy claro, no fue acompañada de condiciones financieras y logísticas que permitieran la ejecución de políticas públicas que impactaran en el sector y así lo deja ver el secretario Montealegre.

La economía nacional durante la primera mitad del siglo XX fue sumamente dependiente de las importaciones para abastecer el mercado interno, así que se importaban productos de primera necesidad para alimentar a la población. En la Tabla 1 se muestran las importaciones de 1927 a 1943, en toneladas para los rubros de arroz, frijoles y maíz.

Desde 1927 a 1943 se pueden observar dos tendencias de comportamiento en la importación de estos productos. El año 1928 marca el máximo volumen de importaciones y a partir de este empieza a declinar hasta 1936 en que, según indica el mismo documento, las tierras de la costa sur del pacífico quedaron acaparadas por el banano (Ministerio de Agricultura e Industria, 1943, p. xviii), y por ende la producción interna decayó de manera importante. De los 3 productos, el más importante y representativo en cuanto a impacto en la nutrición de la población es el arroz y por esto es que presenta las mayores toneladas de importación. El informe además cita al Instituto de asuntos Interamericanos, el cual menciona que: "si Costa Rica dispusiera de todos los otros 
artículos de alimentación, en las cantidades estipuladas, la producción de arroz sería suficiente" (Ministerio de Agricultura e Industria, 1943, p. xviii). Esto quiere decir que el arroz constituye un elemento fundamental en el plato de los costarricenses de la época, que si bien se carece de otros grupos de alimentos, estos requisitos son sustituidos con el arroz.

Tabla 1. Importaciones de 1927-1943 en Toneladas.

\begin{tabular}{|r|r|r|r|r|}
\hline \multicolumn{1}{|c|}{ Año } & \multicolumn{1}{|c|}{ Arroz } & \multicolumn{1}{|c|}{ Frijoles } & \multicolumn{1}{c|}{ Maíz } & \multicolumn{1}{c|}{ Total } \\
\hline 1927 & 2986 & 665 & 1665 & 5316 \\
\hline 1928 & 5270 & 1201 & 5241 & 11712 \\
\hline 1929 & 4310 & 470 & 2511 & 7291 \\
\hline 1930 & 4282 & 26 & 855 & 5163 \\
\hline 1931 & 5132 & 322 & 32 & 5486 \\
\hline 1932 & 1250 & 671 & 65 & 1986 \\
\hline 1933 & 1030 & 22 & 6 & 1058 \\
\hline 1934 & 447 & 47 & 7 & 501 \\
\hline 1935 & 130 & 11 & $-\cdots----$ & 141 \\
\hline 1936 & 448 & 59 & $-\cdots----$ & 507 \\
\hline 1937 & 1400 & 142 & $-\cdots-----$ & 1542 \\
\hline 1938 & 586 & 68 & $-\cdots-----$ & 654 \\
\hline 1939 & 1044 & 779 & 182 & 2005 \\
\hline 1940 & 1174 & 40 & $-\cdots----$ & 1005 \\
\hline 1941 & 1051 & 6 & $-\cdots----$ & 1057 \\
\hline 1942 & 2367 & 3 & 120 & 149 \\
\hline 1943 & 1380 & 1056 & 4803 \\
\hline
\end{tabular}

Fuente: Ministerio de Agricultura e Industria, Memoria 1943.

La necesidad de diversificar la producción nacional fue un rubro fundamental en la política económica de finales de la década de los treinta y principios de los cuarenta, la dependencia del mercado internacional colapsado por la guerra, al igual que una reducción en las exportaciones manifiesta la vulnerabilidad en la que se encontraba la economía del país. No obstante, estos cambios se enfrentaron a varios problemas. Uno de ellos fue lo que denominó Rodrigo Facio como "reglamentismo", término acuñado para referirse a múltiples medidas económicas tomadas en las décadas de los 20 y 30, que 
habían sido aplicadas de una forma aislada una de otra, respondiendo a intereses de grupos de poder, que tuvo como consecuencia la dificultad de establecer políticas coherentes, porque dependiendo del grupo en poder y de sus intereses se le daba o no protección e impulso a algún producto en particular (León, s.a., p. 28).

Otro problema fue el predominio del cultivo del café en la vida económica del país. Todo giraba en torno al café y este tenía un efecto importante en un grueso de la población. Por eso las medidas que se tomaron en torno a la regulación de este producto tenían una significancia palpable a nivel social. Facio argumentaba que la excesiva dependencia del café debido a su alta rentabilidad, desplazaba la producción de otros cultivos menos rentables, pero alimenticios (León, s.a., p. 28).

\section{Requerimientos alimenticios de la población}

En las memorias del Ministerio de Agricultura e Industria de 1943, se presenta un cuadro diseñado por el Instituto de Asuntos Interamericanos, el cual expone los requisitos anuales y del consumo de artículos alimenticios que se producen actualmente o que se pueden producir en Costa Rica y está calculada para dar una alimentación adecuada a la totalidad de la población del país con datos de 1940.

De manera general, lo primero que resalta de este cuadro (ver Tabla 2) es que los requerimientos estimados para la población son mucho mayores que las cantidades consumidas anualmente, esto incluye la producción local y las importaciones. Esto significa que la población no estaba consumiendo los requerimientos nutricionales óptimos para una adecuada salud. Los artículos que se consideraron son alimentos representativos de los diferentes grupos alimenticios y que son fundamentales para una adecuada nutrición.

De acuerdo al primer rubro de la lista, la población costarricense necesitaba para ese entonces 220.000 toneladas de carne o pescado, pero solo consume 12.900 , lo cual evidencia una carencia importante de la proteína animal. El informe que proporciona el Instituto de Asuntos Interamericanos sugiere que: "la acción más inmediatamente efectiva para aumentar la producción de proteína animal, es la expansión de la industria pesquera" (Ministerio de Agricultura e Industria, 1943, p. xiv). De previo el gobierno tiene claro que el país no está ni estará en condiciones de abastecerse por sí mismo de carne, para esto deben promoverse políticas para apoyar al sector en algunos territorios como Guanacaste donde el cultivo de pastos en terrenos, la selección continua de ganado de engorde pueden incidir en mejorar esta carencia.

Tabla 2. Requerimientos anuales y de consumo de artículos alimenticios.

\begin{tabular}{|c|c|c|c|c|}
\hline \multirow{2}{*}{ Artículo } & Cantidad & \multicolumn{3}{|c|}{ Cantidad consumida anualmente } \\
\cline { 3 - 4 } & $\begin{array}{c}\text { requeridas } \\
\text { Toneladas }\end{array}$ & $\begin{array}{c}\text { Producción } \\
\text { local } \\
\end{array}$ & $\begin{array}{c}\text { Importación } \\
\text { Toneladas }\end{array}$ & Total \\
& & & Toneladas & Toneladas \\
\hline
\end{tabular}


Revista Herencia, Vol. 32 (1), enero-junio, 2019.

\begin{tabular}{|c|c|c|c|c|}
\hline Carne y pescado & 220.000 & 12.250 & 650 & 12.900 \\
\hline Leche & 155.000 & 30.000 & 1.500 & 31.500 \\
\hline Huevos & $20.000 *$ & 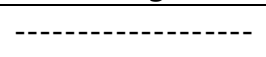 & - & 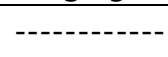 \\
\hline $\begin{array}{l}\text { Papas y raíces } \\
\text { amiláceas }\end{array}$ & $120.000 * *$ & ---------------- & - & 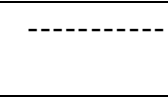 \\
\hline Verduras & 94.000 & 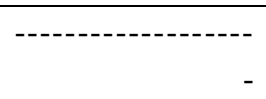 & 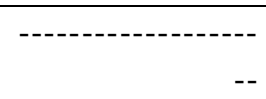 & ----------- \\
\hline Frutas y hortalizas & 59.000 & ------------------- & - & ---------- \\
\hline Grasa o aceite & 8.800 & 800 & 1.450 & 2.250 \\
\hline Arroz & 22.000 & 21.400 & 1.100 & 22.500 \\
\hline Maíz & 18.000 & 37.200 & 300 & 37.500 \\
\hline Frijoles & 27.000 & 12.000 & 500 & 12.500 \\
\hline Dulce & 25.000 & 31.150 & 2.000 & 33.150 \\
\hline $\begin{array}{l}\text { Aceite de hígado de } \\
\text { pescado }\end{array}$ & 21 & 17 & 2 & 19 \\
\hline
\end{tabular}

Fuente: Ministerio de Agricultura e Industria, Memoria 1943.

La producción de leche también se encuentra disminuida si se compara con los requerimientos estimados. Se consumen 31.500 toneladas, pero se deberían consumir 155.000 toneladas, esta diferencia importante es de sumo interés, pues como lo indica el documento, este producto es indispensable para la raza (Ministerio de Agricultura e Industria, 1943, p. xiv). De acuerdo a esta proyección, indica el Ministro:

Se requiere aumentar cinco veces la industria lechera si queremos, como es nuestro deber, criar niños sanos y fuertes y aminorar un tanto la mortalidad infantil que no hace, por cierto, honor a la cultura del país. (Ministerio de Agricultura e Industria, 1943, p. xiv)

Con respecto al arroz, vemos que es el único artículo en donde lo que se consume coincide con los requerimientos, 22.000 toneladas es la estimación y 22.500 toneladas es lo que se consume. Esto se correlaciona con la Tabla 1 en donde el Instituto de Asuntos Interamericanos mencionaba que de consumirse el resto de productos, la cantidad de arroz que se producía era suficiente para cubrir las cantidades básicas de consumo.

La atención que merecía para los gobiernos de turno la nutrición de la población es evidente, no solo por medio del Instituto de Asuntos Interamericanos o el Consejo Nacional de Nutrición, sino que también a través del apoyo al sector agrícola se buscaba dotar al mercado interno de buena parte de los alimentos de primera necesidad. Es así como se implementaban estudios para determinar el potencial de producción. Por ejemplo, en 1948 la revista Suelo Tico publica en la edición de diciembre un censo de todos 
los trapiches que se encontraban en el territorio nacional con el objetivo de determinar el cultivo de la caña de azúcar y producción de dulce y panela.

Tabla 3. Número de trapiches por provincia en 1948.

\begin{tabular}{|c|c|c|}
\hline Provincias & & Trapiches \\
\hline San José & 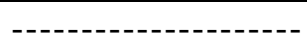 & 1036 \\
\hline Alajuela & - & 415 \\
\hline Cartago & 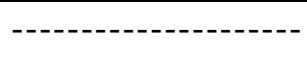 & 167 \\
\hline Heredia & 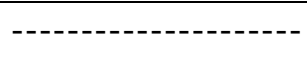 & 44 \\
\hline Guanacaste & 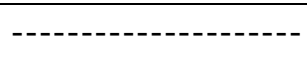 & 274 \\
\hline Puntarenas & 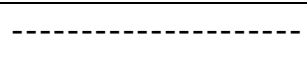 & 212 \\
\hline Limón & 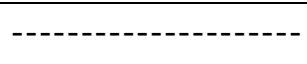 & 27 \\
\hline Total & - & 2175 \\
\hline
\end{tabular}

Fuente: Revista Suelo Tico, Vol1, No 5 , diciembre 1948.

La mayoría de los trapiches se concentran en San José, seguido de Alajuela y Cartago, en el valle central, esto explica el abastecimiento interno de los productos derivados de esta pequeña industria como lo eran el azúcar, el dulce y la panela. Las 3 provincias periféricas presentan una modesta pero importante cantidad, siendo Limón el que presenta la menor. Esto también se explica por la concentración de la población en donde los centros urbanos más densos, poblacionalmente hablando, se encuentran en el interior del país.

El censo abarcó, no solo la cantidad de trapiches por provincia, sino que también se evaluó la cantidad de pailas que había en cada uno y la cantidad de galones que pueden contener cada una de ellas, con esto se podía determinar la capacidad de producción. Por otro lado, se registraron los quintales de panela y dulce por semana y durante todas las semanas de trabajo, lo que nos da una idea de la producción de estos bienes. La información se presenta en la Tabla 4 .

Como se observa, la información en cuanto a la producción de los trapiches que fueron censados en 1948 incluye además de determinar su distribución por provincias el registró la producción en quintales por semana de dulce, panela y ambos; incluso calcula la totalidad de quintales producidos al final de todas las semanas trabajadas. Esto es de 
suma importancia para documentar la producción nacional, la disponibilidad de la misma y los requerimientos satisfechos desde las proyecciones que se generaban para la población. Por ejemplo, para el caso de Cartago que cuenta con 167 trapiches, representa un $\mathbf{1 7 \%}$ de la totalidad de la producción nacional. La provincia que asume la mayor producción de dulce y panela es Alajuela, con un 40\% y la menor es Limón que no muestra ningún porcentaje representativo a escala nacional. Como mencionábamos en el análisis de la tabla anterior, la mayor concentración se encuentra en el Valle Central. Esta información servía de insumo al Concejo Nacional de Nutrición para realizar las proyecciones correspondientes para la población. 
Tabla 4. Número de trapiches, producción de dulce y panela por semana y en total de semanas trabajadas durante el año 1948.

\begin{tabular}{|c|c|c|c|c|c|c|c|c|}
\hline Provincia & $\begin{array}{l}\text { Número } \\
\text { de } \\
\text { trapiches }\end{array}$ & $\begin{array}{l}\text { Quintales } \\
\text { de dulce } \\
\text { por } \\
\text { semana }\end{array}$ & $\begin{array}{l}\text { Quintales } \\
\text { de panela } \\
\text { por } \\
\text { semana }\end{array}$ & $\begin{array}{l}\text { Total de } \\
\text { quintales de } \\
\text { dulce y } \\
\text { panela por } \\
\text { semana }\end{array}$ & $\begin{array}{l}\text { Producción } \\
\text { quintales de } \\
\text { panela en } \\
\text { total en } \\
\text { semana }\end{array}$ & $\begin{array}{l}\text { Producción de } \\
\text { quintales de } \\
\text { dulce por } \\
\text { semana }\end{array}$ & $\begin{array}{l}\text { Producción de } \\
\text { quintales de } \\
\text { dulce y panela } \\
\text { por semana }\end{array}$ & $\begin{array}{c}\% \text { de } \\
\text { producción de } \\
\text { cada provincia } \\
\text { en relación con } \\
\text { el país }\end{array}$ \\
\hline San José & 1.036 & 3.174 & 724 & 3.898 & 134.843 & $34 \cdot 330$ & 169.173 & 23 \\
\hline Alajuela & 415 & 5.878 & 702 & 6.681 & 267.382 & 31.858 & 299.240 & 40 \\
\hline Cartago & 167 & 2.823 & 42 & 2.866 & $125 \cdot 53^{8}$ & 1.827 & 127.365 & 17 \\
\hline Heredia & 44 & 999 & 2 & 1.101 & 46.057 & 112 & 46.169 & 6 \\
\hline Guanacaste & 274 & 1.419 & 7 & 1.427 & 60.396 & 285 & 60.881 & 8 \\
\hline Puntarenas & 212 & 548 & ------------ & 548 & 26.334 & 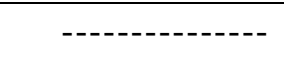 & 26.334 & 3 \\
\hline Limón & 27 & 58 & 40 & 98 & 2.559 & 1.760 & $4 \cdot 319$ & 0 \\
\hline Totales & 2.175 & 14.901 & 1.519 & 16.521 & 663.112 & 70.173 & $733 \cdot 485$ & 100 \\
\hline
\end{tabular}

Fuente: Revista Suelo Tico, Vol 1, № 5, diciembre 1948. 


\section{La atención del sector agrícola}

Es evidente que el fortalecimiento de la producción agrícola local constituye un factor que repercute en el abastecimiento y consumo de la población. Es por eso que el Ministerio de Agricultura e Industria ejecuta una serie de programas para apoyar al sector, entre estos contar con la asesoría de STICA: Servicio Técnico Inter-Americano de Cooperación Agrícola, del cual la mayoría de labores estaban orientadas en mejorar la conducción de los diferentes cultivos (Ministerio de Agricultura e Industria, 1951, p. 178).

La extensión agrícola que proyectaba STICA buscaba, además de la liberación económica de la población campesina con la promoción de más y mejores cosechas, el mejoramiento del hogar y las comunidades rurales, en todos los aspectos: "no solo de la producción y distribución agrícola, sino también de la elevación de los niveles de la salud y la nutrición" (Ministerio de Agricultura e Industria, 1949, p.32).

Otro organismo que logró instaurarse en las comunidades fueron los Clubes 4-S (salud, servicio, sentimiento y saber), asociaciones de jóvenes que se reunían para ejecutar proyectos de agricultura, de cría de animales o de otra industria y de colaborar en todo aquello que significara progreso comunal y dignificación de la vida en el campo (Ministerio de Agricultura e Industria, 1949, p.32).

Durante buena parte de la primera mitad del siglo $\mathrm{XX}$, las políticas gubernamentales sobre nutrición empezaron a dar los primeros pasos sobre el interés de proteger el derecho de la población a una alimentación digna. En la década de los cuarentas se logra observar algunos esfuerzos, que se ampliarán en las siguientes décadas a medida que el concepto de seguridad alimentaria y salud de la población se consolida.

\section{Los principales organismos internacionales e instituciones nacionales: Seguridad Alimentaria y Nutricional desde los años cincuenta y sesenta en Costa Rica}

\section{La Sociedad de La Gota de Leche}

Basándonos en la información provista por William Vargas en la obra que él colaboró llamado La transición de la Nutrición y la Salud de Costa Rica explica que desde los inicios de los años treinta se habían observado graves casos de proteína-calórica. Se explica en esa publicación que uno de los primeros programas que intentaban reducir la nutrición proteino-calórica fue el programa Costarricense de Alimentación Complementaria para niños menores de seis años de edad, escolares y mujeres embarazadas (Muñoz y Scrimshaw, 1996, p. 101). Ahora bien, existieron programas anteriores del programa Costarricense de Alimentación. Hay documentación de una organización benéfica llamada Sociedad de la Gota de Leche. Una asociación que estaba destinada a mejorar la salud de los niños, cuyo centro estaba en la ciudad de San José en 1913. Citando a Virginia Mora (en Viales, 2005), la Gota de Leche josefina -que se expande hacia Alajuela y Heredia en 1914-, no es solo brindar alimentos a las y los niños pobres, sino también educar a las madres en lo relativo a la mejor crianza de sus hijos e hijos, como lo manifiesta 
el hecho de que se nombrara como médica de esta institución, a la doctora Jadwisia de Picado, la cual muestra particular interés por la salud de las madres. Dentro de este creciente interés por la protección materna e infantil, cada vez adquiere mayor vigencia pública el tema de la importancia de la leche materna (Viales, 2005, pp. 87-88).

Se sabe que hubo un libro de registro o el Diario de la Gota de Leche, el cual abarca desde el 14 de octubre de 1947 hasta el 10 de junio de 1952. Se proveían Botellas del 1950 al 1952 de que oscilan $53-88$ unidades, para un total de 90 niños. Una sociedad que se financiaba gracias a las donaciones, que llegaban cada 15 días (CR-AN-CR, Fondo SGLESHE, Signatura 000008). Respecto a ese punto aquí hay una lista de los mayores donantes hacia esa sociedad.

Gráfico 1. Donantes y cantidad de colones durante los años 1947 a 1952.

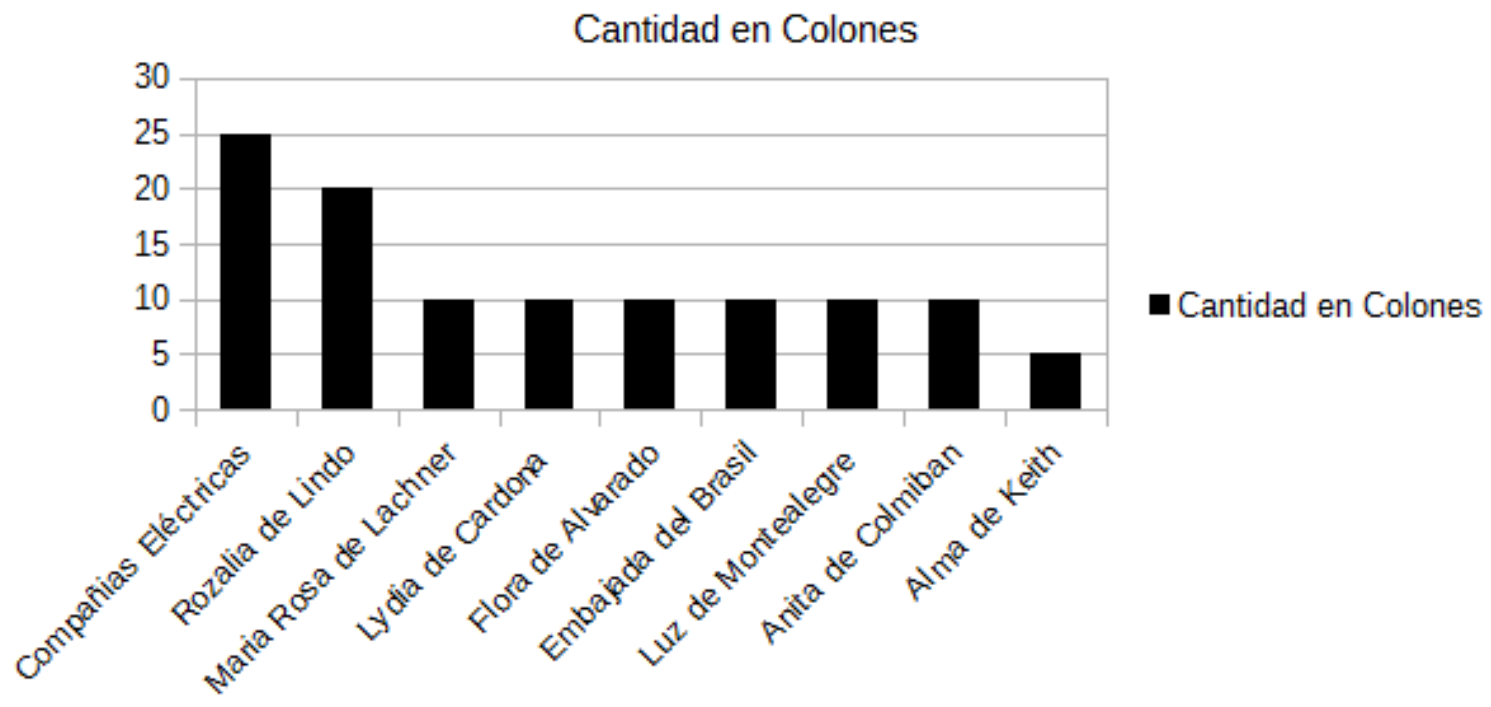

Fuente: CR-AN-AH. Fondo: SGLECHE. Signatura. 000008.

Se puede observar que el mayor contribuyente fueron las compañías de electricidad (que no se sabe por cierto el motivo, si es que hubiere) con un valor de 25 colones cada quince días, luego le sigue Rozalia de Lindo. Ahora bien, un actor destacado en este cuadro es la Embajada de Brasil. Se sabe que en ese mismo país suramericano se había realizado $A X I$ Conferencia Sanitaria Panamericana en Rio de Janeiro el 6 de septiembre de 1942, ahí estuvo presente Costa Rica. Se sabe que en esa conferencia se le dio particular importancia el tema de la nutrición y el acceso de la leche en la infancia (CR-AN-AH, Fondo MRREE, Signatura 16397). Este caso qué nos quiere decir, bueno que los intentos de colaboración internacional a raíz de solventar el problema de la desnutrición son anteriores al tema de investigación.

La sociedad de la Gota de Leche se mantuvo activa desde los años de 1913 hasta su aparente clausura en 1959, las razones de tal clausura no se pudieron explicar, sin 
embargo habría que preguntarse si su impacto fue tan significativo para decir que fue un mecanismo efectivo para combatir los niveles de nutrición. Para ello, se pudo encontrar la cantidad de leche comprada y la cantidad de niños que manejaban cada mes en una serie de años: 1953, 1956, 1957 y 1958.

Tabla 5. Instancias de enfermos gratuitos, 1953. Consumo anual de leche categorizado por mes según Dirección General de Asistencia Medico Social-Sección Hospitalaria.

\begin{tabular}{|c|c|c|c|c|c|c|}
\hline \multirow{2}{*}{ Fecha } & \multirow{2}{*}{$\begin{array}{l}\text { Cantidad de } \\
\text { Botellas }\end{array}$} & \multirow{2}{*}{ Unidad } & \multirow{2}{*}{ Precio } & \multicolumn{3}{|c|}{$\begin{array}{c}\text { Estancias de Enfermos } \\
\text { Gratuitos }\end{array}$} \\
\hline & & & & $\begin{array}{c}\text { Q } \\
\text { Varones }\end{array}$ & $\begin{array}{c}\text { Q } \\
\text { Mujeres }\end{array}$ & Total \\
\hline diciembre & 2945 & 0,55 & $\mathbb{L}_{1} 619,75$ & 47 & 43 & 92 \\
\hline noviembre & 2850 & & $\mathbb{L}_{1567,50}$ & 47 & 45 & 92 \\
\hline septiembre & 2945 & & $\mathbb{L}_{1} 619,75$ & 45 & 45 & 90 \\
\hline agosto & 2945 & & $\mathbb{L}_{1} 619,75$ & 47 & 45 & 92 \\
\hline julio & 2931 & & $\mathbb{L}_{1612,05}$ & 45 & 47 & 92 \\
\hline junio & 2728 & & $\mathbb{W}_{1500,40}$ & 47 & 43 & 90 \\
\hline enero & 2790 & & $\mathbb{C}_{1534,50}$ & 47 & 43 & 90 \\
\hline febrero & 2464 & & $\mathbb{L}_{1355,20}$ & 43 & 43 & 86 \\
\hline marzo & 2728 & & $\mathbb{W}_{1500,40}$ & 44 & 42 & 86 \\
\hline abril & 2640 & & $\mathbb{L}_{1452,00}$ & 43 & 43 & 86 \\
\hline
\end{tabular}

Fuente: CR-AN-AH. Fondo: SGLECHE. Signatura. 000008.

Tabla 6. Instancias de enfermos gratuitos, 1956-1957. Consumo anual de leche categorizado por mes según Dirección General de Asistencia Médico Social.

\begin{tabular}{|c|c|c|c|c|c|c|c|}
\hline \multirow{2}{*}{ Año } & \multirow{2}{*}{ Fecha } & \multirow{2}{*}{$\begin{array}{l}\text { Cantidad } \\
\text { de } \\
\text { Botellas }\end{array}$} & \multirow{2}{*}{ Unidad } & \multirow{2}{*}{ Precio } & \multicolumn{3}{|c|}{$\begin{array}{c}\text { Estancias de Enfermos } \\
\text { Gratuitos }\end{array}$} \\
\hline & & & & & $\begin{array}{c}\text { Q } \\
\text { Varones }\end{array}$ & $\begin{array}{c}\mathrm{Q} \\
\text { Mujeres }\end{array}$ & Total \\
\hline 1956 & agosto & 2790 & 0,6 & $\mathbb{L}_{1} 674,00$ & 44 & 44 & 88 \\
\hline 1956 & julio & 2790 & & $\mathbb{L}_{1} 674,00$ & 47 & 43 & 90 \\
\hline 1956 & mayo & 2790 & & $\mathbb{L}_{1} 674,00$ & 44 & 44 & 88 \\
\hline 1956 & junio & 2700 & & $\mathbb{L}_{1620,00}$ & 44 & 44 & 88 \\
\hline 1956 & abril & 2790 & & $\mathbb{L}_{1} 674,00$ & 43 & 45 & 88 \\
\hline 1956 & marzo & 2790 & & $\mathbb{L}_{1} 674,00$ & 45 & 43 & 88 \\
\hline 1956 & febrero & 2610 & & $\mathbb{L}_{1566,00}$ & 45 & 43 & 88 \\
\hline 1956 & enero & 2790 & & $\mathbb{L}_{1674,00}$ & 44 & 44 & 88 \\
\hline 1956 & diciembre & 2790 & & $\mathbb{L}_{1} 674,00$ & 47 & 43 & 90 \\
\hline 1956 & octubre & 2728 & & $\mathbb{L}_{1} 636,80$ & 45 & 43 & 88 \\
\hline
\end{tabular}


Revista Herencia, Vol. 32 (1), enero-junio, 2019.

\begin{tabular}{|c|c|c|c|c|c|c|}
\hline 1956 & septiembre & 2640 & $\mathbb{L}_{1584,00}$ & 45 & 43 & 88 \\
\hline 1957 & diciembre & 2790 & $\mathbb{L}_{1674,00}$ & 45 & 45 & 90 \\
\hline 1957 & febrero & 2520 & $\mathbb{L}_{1512,00}$ & 45 & 45 & 90 \\
\hline 1957 & enero & 2790 & $\mathbb{L}_{1674,00}$ & 45 & 45 & 90 \\
\hline 1957 & marzo & 2790 & $\mathbb{L}_{1} 674,00$ & 46 & 44 & 90 \\
\hline 1957 & abril & 2700 & $\mathbb{L}_{1} 620,00$ & 45 & 45 & 90 \\
\hline 1957 & mayo & 2790 & $\mathbb{W}_{1} 674,00$ & 47 & 43 & 90 \\
\hline 1957 & junio & 2640 & $\mathbb{L}_{1584,00}$ & 45 & 45 & 90 \\
\hline 1957 & julio & 2790 & $\mathbb{L}_{1} 674,00$ & 42 & 48 & 90 \\
\hline 1957 & agosto & 2790 & $\mathbb{L}_{1674,00}$ & 46 & 44 & 90 \\
\hline 1957 & setiembre & 2700 & $\mathbb{L}_{1} 620,00$ & 47 & 43 & 90 \\
\hline 1957 & octubre & 2790 & $\mathbb{L}_{1} 674,00$ & 45 & 45 & 90 \\
\hline 1957 & noviembre & 2700 & $\mathbb{L}_{1620,00}$ & 45 & 45 & 90 \\
\hline
\end{tabular}

Fuente: CR-AN-AH. Fondo SGLECHE oooog1.

Tabla 7. Instancias de enfermos gratuitos, 1958. Consumo anual de leche categorizado por mes según Dirección General de Asistencia Médico Social.

\begin{tabular}{|c|c|c|c|c|c|c|c|}
\hline \multirow{2}{*}{ Año } & \multirow{2}{*}{ Fecha } & \multirow{2}{*}{$\begin{array}{l}\text { Cantidad } \\
\text { de } \\
\text { Botellas }\end{array}$} & \multirow{2}{*}{$\begin{array}{l}\text { Unida } \\
\text { d }\end{array}$} & \multirow{2}{*}{ Precio } & \multicolumn{3}{|c|}{$\begin{array}{c}\text { Estancias de Enfermos } \\
\text { Gratuitos }\end{array}$} \\
\hline & & & & & $\begin{array}{c}\text { Q } \\
\text { Varones }\end{array}$ & $\begin{array}{c}\text { Q } \\
\text { Mujeres }\end{array}$ & Total \\
\hline \multirow[t]{10}{*}{1958} & Marzo & 2790 & 0,6 & $\mathbb{1} 1674$ & 45 & 45 & 90 \\
\hline & abril & 2700 & & $\mathbb{4} 1620$ & 47 & 43 & 90 \\
\hline & mayo & 2790 & & $\mathbb{1} 1674$ & 45 & 45 & 90 \\
\hline & junio & 2700 & & $\mathbb{1} 1620$ & 48 & 42 & 90 \\
\hline & julio & 2790 & & $\mathbb{1} 1674$ & 45 & 45 & 90 \\
\hline & agosto & 2700 & & $\mathbb{1} 1620$ & 47 & 43 & 90 \\
\hline & septiembre & 2790 & & $\mathbb{L} 1674$ & 45 & 45 & 90 \\
\hline & octubre & 2790 & & $\mathbb{1} 1674$ & 45 & 45 & 90 \\
\hline & noviembre & 2700 & & $\mathbb{4} 1620$ & 47 & 43 & 90 \\
\hline & diciembre & 2785 & & $\mathbb{1} 1671$ & 45 & 45 & 90 \\
\hline
\end{tabular}

Fuente: CR-AN-AH. Fondo Salud. Signatura 212.

En las Tablas 5, 6 y 7 vemos, en un lapso interrumpido de cuatro años, que se sirvió a unos 3848 , lo cual parece ser una iniciativa muy significativa, sin embargo, el promedio desde 
1953 hasta 1958 estuvo siempre ocupada aproximadamente por 90 niños. Eso quiere decir que la cantidad no varió, ni disminuyó ni aumentó el número de niños atendidos.

La Sociedad de la Gota de Leche es un ejemplo claro de como asociaciones benéficas pueden de alguna manera colaborar en la nutrición especialmente la infantil. Ahora bien, cabe destacar que la primera encuesta de nutrición de 1966 realizada a nivel nacional mostró que un $57 \%$ de los niños mostraban algún grado de desnutrición, un 18\% de los escolares padecían de bocio endémico (falta de proteínas calóricas y yodo), un 32,5\% mostraban deficiencia de retino sérico y se tenían casos de anemia en madres embarazadas, lo cual se debe a una falta de hierros y folatos (Muñoz y Scrimshaw, 1996, p. 101). Eso explica que los programas anteriores a ese años de 1966 no eran tan significativos.

\section{Los Programas de las Naciones Unidas y el Gobierno de Costa Rica}

A los inicios de los años 50 se comienzan a hacer trabajos en obra pública que buscaban crear centros o albergues especializados en proveer de un paquete alimenticio a los niños con déficit nutricional, estos recintos se llaman Centros de Nutrición. El primero se ubicó en Barva de Heredia y para los años de 1950 y 1960 ya estaban en las principales provincias, sin embargo se quería llevar a cabo un plan para ubicarlos en zonas rurales (Muñoz y Scrimshaw, 1996, pp. 101-103).

La diferencia entre la cantidad de personas con problemas nutricionales entre población urbana o rural no se ha podido saber con exactitud, sin embargo, sí se ha podido saber qué es lo que comían. Según el proyecto del Gobierno de Costa Rica y el Programa Mundial de Alimentos (PMA) sobre Nutrición Aplicada, el campesino tenía un alto consumo de azúcar, leguminosas y granos, legumbres y frutas pero era ocasional el consumo de carne y leche (CR-AN-AH, Fondo MRREE, Signatura 8822, Folio 11). Este programa implementado en 1966 trataba suplirles con un paquetes de alimentos que consistían en lo siguiente:

\footnotetext{
Harina de trigo y maíz, 200 gramos. Leche en polvo 50 gramos, carne enlatada 30 gramos, huevos deshidratados 5 gramos, aceite vegetal o manteca de cerdo 30 gramos, fruta seca 20 gramos, pecado enlatado 20 gramos, total 555 gramos de alimentos. (CR-AN-AH, Fondo MRREE, Signatura 8822, Folio 12-13) ${ }^{1}$
}

Es oportuno aclarar que los organismos de corte internacional: el PMA (Programa Mundial de Alimentos), la FAO (La Organización de las Naciones Unidas para la Alimentación y la Agricultura), y la UNICEF (El Fondo de las Naciones Unidas para la Infancia) junto con diferentes entes gubernamentales como el MAG (Ministerio de Agricultura y Ganadería), Ministerio de Salud, el Ministerio de Educación Pública y la

\footnotetext{
${ }^{1}$ Cabe destacar que este programa era bianual. El primer año se esperaba que se beneficiasen 5 mil, en el segundo 10 mil, y en el último 5 mil más. El tamaño medio familiar es 6 , en total 60 mil beneficiados. Ayuda alimentario por un total de 730 días por familia, la llegada debería ser cada 3 meses y la distribución para 15 días.
} 
Oficina de Planificación, tenían una serie de objetivos para mejorar la distribución, producción y el consumo de alimentos a nivel nacional:

1. Crear una red de acueductos en zonas rurales para solventar el Plan Decenal de Salud Pública de la Alianza para el Progreso, suministrar como mínimo, agua potable al 50\% de la población rural de América Latina. Pero que también estaba sustentando la seguridad alimentarias de la zona (CR-AN-AH, Fondo MRREE, Signatura 8822, Folio 96) ${ }^{2}$.

2. Proveer de materiales fundamentales para que las zonas rurales puedan autoabastecerse mediante la otorgación de hectáreas cultivables e insumos, específicamente de emergencia (CR-AN-AH, Fondo MRREE, Signatura 8822, Folio 5) ${ }^{3}$.

3. Crear una red de distribución efectivo de raciones y paquetes nutricionales.

Sobre el primer objetivo, el de la construcción de los acueductos en zonas rurales, se pudo evidenciar el impacto que este tuvo sobre los trabajadores y las comunidades seleccionadas. Con el propósito de mostrar su relevancia, se generaron dos tablas (8 y 9), una respecto al proceso de construcción de acueductos y el otra respecto a la cantidad de alimentos por constructores de los acueductos en distintas zonas rurales.

Estas dos tablas (ver Tablas 8 y 9) evidencian la creación de los acueductos. Ahora bien, esta política de obra pública estaba sustentada por dos condiciones, los trabajadores se les debía pagar un salario monetario, pero complementándoles con un paquete de alimentos. Esto se hizo porque el ingreso del trabajador era muy bajo, la tarifa se ajustaría no al nivel de la construcción del acueducto, sino a las condiciones económicas de los campesinos (CR-AN-AH, Fondo MRREE, Signatura 8822, Folio 46)4. La participación de las comunidades se obtendría por diversos canales a saber: mano de obra, aporte monetario, transportes, materiales, etc. Una vez terminado el acueducto, los vecinos participarían en la administración y la operación del mismo a partir de una junta administradora.

\footnotetext{
2 Este trabajo se realiza en conjunto con la SNAA (Sistema Nacional De Acueductos y Alcantarillados).

3 Por ejemplo en 1967 el Volcán Tilarán había hecho erupción, así que hubo ayuda del PMA en comunidades de Guanacaste y Tillarán, el cual consistía en dos cosas, la primera era un proyecto para ayudar a 10 mil familias, en las áreas devastadas en las provincias de Guanacaste y Alajuela, aprovechando la ayuda alimentaria se harán trabajos en una área aprox. De 30 mil hectáreas, se espera en que trabajen en 500 hectáreas en riego , 500 en reparación y en anchura de cercas, 50 mil metros cuadrados en habitaciones y galeras, 10 mil metros en gallineros, galerones, 6 mil hectáreas de conservación de suelos, 100 kilómetros de nuevas cañerías, 50 kilómetros de caminos.

4 Algo que es importantísimo destacar es una vez terminado el acueducto, los vecinos participarán en la administración y la operación del mismo a partir de una junta administradora. Esto puede significar el inicio de lo administración regional de las ASADAS.
} 
Tabla 8. Puntos de distribución de alimentos, 1967.

\begin{tabular}{|c|c|c|c|}
\hline $\begin{array}{l}\text { Distribución alimentos por la } \\
\text { construcción de las cañerías }\end{array}$ & Primer Reparto & Último reparto & $\begin{array}{l}\text { Terminado } \\
\text { o no }\end{array}$ \\
\hline $\begin{array}{l}\text { Belén- Santa Ana- Santo } \\
\text { Domigo }\end{array}$ & 8 de enero & 30 de setiembre & $\mathrm{Si}$ \\
\hline Sardinal & 8 de enero & 30 de setiembre & $\mathrm{Si}$ \\
\hline Buenos Aires & 8 de enero & 30 de setiembre & \\
\hline Villa Neily & 8 de enero & 30 de setiembre & \\
\hline Lagunilla & 8 de enero & 28 de mayo & \\
\hline Matina & 8 de enero & 28 de mayo & \\
\hline Estrada & 8 de enero & 28 de mayo & \\
\hline La Cruz & 8 de enero & 23 de julio & $\mathrm{Si}$ \\
\hline Guardia Comunidad & $8 \mathrm{~d}$ eenro & 17 de setiembre & \\
\hline $\begin{array}{l}\text { San Ramón Norte-San Ramón } \\
\text { Sur-Pedregositos }\end{array}$ & $8 \mathrm{~d}$ eenro & 30 de setiembre & \\
\hline Rio Cañas & 22 de enero & 23 de julio & $\mathrm{Si}$ \\
\hline La Fortuna & 19 de febrero & 20 de agosto & $\mathrm{Si}$ \\
\hline Peñas Blancas & 30 de abril & 30 de setiembre & \\
\hline Cedral-Puente Casa Colón & 30 de abril & 30 de setiembre & \\
\hline Tinamaste-Alfombra & 30 de abril & 30 de setiembre & \\
\hline San Francisco-Caimitos & 30 de abril & 30 de setiembre & \\
\hline Pénjamo (San Carlos) & 30 de abril & 6 de agosto & $\mathrm{Si}$ \\
\hline Atenas & 17 de septiembre & 30 de septiembre & \\
\hline Santa Bárbara-Oriente & 30 de setiembre & 30 de septiembre & \\
\hline 27 de abril & 30 de septiembre & 30 septiembre & \\
\hline
\end{tabular}

Fuente: Cuadro incorporado en los folios 37 y 38 del fondo MRREE, Signatura 8822.

Tabla 9. Resumen de los alimentos distribuidos entre el 5 de abril de 1967 y el 25 de agosto de 1968. 


\begin{tabular}{|c|c|c|c|c|c|c|c|}
\hline Proyecto & $\begin{array}{c}\text { Maíz } \\
\text { (libras) }\end{array}$ & $\begin{array}{c}\text { Leche } \\
\text { (paquetes) }\end{array}$ & $\begin{array}{l}\text { Aceite } \\
\text { (latas) }\end{array}$ & $\begin{array}{l}\text { Carne } \\
\text { (latas) }\end{array}$ & $\begin{array}{l}\text { Queso } \\
\text { (latas) }\end{array}$ & $\begin{array}{l}\text { Harina } \\
\text { (libras) }\end{array}$ & Raciones \\
\hline $\begin{array}{l}\text { Buenos Aires de } \\
\text { Puntarenas }\end{array}$ & 4900 & 204 & 95 & 849 & & 2200 & 105,5 \\
\hline Villa Neily de Puntarenas & 11800 & 540 & 235 & 2448 & & 6200 & 230 \\
\hline Lagunilla de Santa Cruz & 550 & 26 & 13 & 129 & & 350 & 12 \\
\hline La Cruz de Liberia & 5300 & 218 & 91 & 1080 & & 2700 & 99 \\
\hline La Guardia de Liberia & 2950 & 122 & 49 & 609 & & 1650 & 46,5 \\
\hline San Ramón Perez Zeledón & 4900 & 192 & 91 & 864 & & 2500 & 92,5 \\
\hline La Fortuna de San Carlos & 2500 & 120 & 30 & 504 & & 1200 & 57,5 \\
\hline $\begin{array}{l}\text { Peñas Blancas de Perez } \\
\text { Zeledón }\end{array}$ & 8800 & 378 & 168 & 1824 & & 4300 & 174 \\
\hline $\begin{array}{l}\text { Dulce Nombre de San } \\
\text { Carlos }\end{array}$ & 19600 & 808 & 270 & 3822 & 14 & 8550 & 378 \\
\hline $\begin{array}{l}\text { Tinamaste y Alfombra de } \\
\text { Pérez Zeledón }\end{array}$ & 8000 & 372 & 163 & 1704 & & 4000 & 156,5 \\
\hline Florencia de San Carlos & 17250 & 712 & 227 & 3610 & & 7800 & 352 \\
\hline Pénjamo de San Carlos & 4700 & 192 & 72 & 936 & & 2400 & 93 \\
\hline Belen de Carrilo & 2600 & 120 & 54 & 612 & & 1600 & 39 \\
\hline Sardinal de Carrillo & 1600 & 72 & 36 & 336 & & 1000 & 28,5 \\
\hline Atenas & 23240 & 939 & 189 & 4119 & 153 & 5350 & 414 \\
\hline Santa Bárbara de Sta Cruz & 3506 & 156 & 36 & 720 & & 1100 & 62,5 \\
\hline 27 de abril de Santa Cruz & 3800 & 150 & 30 & 834 & & 1200 & 75,5 \\
\hline Pejivalle de Perez Zeledón & 6700 & 254 & 45 & 1253 & 146 & 3350 & 131,5 \\
\hline $\begin{array}{l}\text { General Viejo y Hermosa } \\
\text { de Perez Zeledon }\end{array}$ & 5100 & 162 & 58 & 993 & 105 & 2550 & 100 \\
\hline $\begin{array}{l}\text { Santa Elena y Quizarrá de } \\
\text { Perez Zeledon }\end{array}$ & 3900 & 154 & 25 & 770 & 78 & 1950 & 77 \\
\hline $\begin{array}{l}\text { Santa Teresa y Cajón de } \\
\text { Perez Zeledon }\end{array}$ & 2400 & 93 & 22 & 555 & 57 & 1200 & 47 \\
\hline Totales & 144096 & 5984 & 1999 & 28571 & 553 & 63150 & 2771,5 \\
\hline
\end{tabular}

Fuente: Cuadro incorporado el folio 42 del fondo MRREE, Signatura 8822.

El costo total del proyecto fue de 22850000 colones. El financiamiento de este proyecto también fue multilateral. Por ejemplo: la Población beneficiada debía pagar 1350000 colones (6\%), el Gobierno Central unos $\mathbb{\$} 2700000$ (12\%), el SNAA (Sistema Nacional de Acueductos y Alcantarillados) $\mathbb{W} 9450000$ (41\%) Banco Interamericano de Desarrollo (Empréstito para el SNNA) $\mathbb{\$} 6650000$ (29\%), PMA Alimentos (valorados) $\mathbb{L}_{2} 000000$ (9\%) Y PMA Aporte en efectivo $\mathbb{W} 700000(0,3 \%)(C R-A N-A H$, Fondo MRREE, Signatura 8822, Folio 46).

No sólo hubo una colaboración multilateral a raíz del financiamiento sino también a raíz de la distribución (CR-AN-AH, Fondo MRREE, Signatura 8822, Folio 36-37). También se identificaron dos puntos de almacenamiento. 
1. Consejo Nacional de Producción, bodegas y cámaras de refrigeración, San José Centro. Llegaban desde Puntarenas, los servicios del Ferrocarril Eléctrico al Pacífico y Vehículos de la Institución.

2. San José Pavas bodega de la oficina de planificación. (CR-AN-AH, Fondo MRREE, Signatura 8822, Folio 36-37).

\section{El programa de Educación Nutricional y Huertos. Las dos etapas: 1966-1967, 1968-} 1969

La política de seguridad alimentaria tenía una naturaleza integradora. No sólo con la incorporación de sistemas de acueductos, de raciones alimentarias o promover autoabastecimiento rural, sino también generar un impacto en la estructura escolar. William Vargas explica que existió un programa llamado Nutrición Familiar y Jardín Escolar. Estaba enfocado en la producción de alimentos en la escuela y en los hogares. Promover una mejor utilización de los alimentos. Promover la educación nutricional de la escuela, mejorar los hábitos alimenticios en la escuela y para las madres (Muñoz y Srimshaw, 1996, p. 104). Este programa también tenía el nombre de Plan de Nutrición y Huertos y era aplicado a los muchachos de sexto grado, en varias escuelas, muchas de ellas de carácter rural (CR-AN-AH, Fondo Ministerio de Educación Pública, Signatura 5056). Funcionaban como una espacie de subciclo lectivo, donde llegaban profesores contratados por el MEP para aplicar las clases.

Cabe destacar la importancia de los centros de nutrición porque eran los espacios donde se proveía los paquetes alimenticios y la atención de las madres. Este proyecto consistía en dos etapas: uno que abarca 1966 a 1967, y la otra (con mayor dimensión) de 1968-1969. En la primera etapa se tomaron 37 escuelas de las cuatro provincias centrales, se atendieron a unos 11093 estudiantes y se tomaron en cuenta 19 Centros de Nutrición: Filadelfia Santa Cruz Bagaces, Tilarán, San Joaquín de Flores San Rafael de Heredia, Santo Domingo de Heredia, Heredia, Sabanilla, Poás, Naranjo, San Ramón, Pérez Zeledón, Paraíso y Orosi (CR-AN-AH, Fondo MREE. Signatura 8527).

En la segunda fase, la de 1968 y 1969, se tomaron 60 centros estudiantiles que provenían de cinco provincias: Cartago, Heredia, San José, Alajuela y Puntarenas. Se atendieron 11737 alumnos y unos 48 Centros de Nutrición se tomaron en cuenta (CR-AN-AH, Fondo MREE. Signatura 8527).

\section{Discusiones sobre la situación alimentaria del país en la revista del Ministerio de Agricultura e Industria de Costa Rica: Suelo Tico}

Para un mejor conocimiento de los procesos de innovación técnica, tecnológica y el entendimiento de las limitaciones socio-ambientales o biológicas de la actividad agroalimentaria según las preocupaciones y misceláneas del pasado, podemos ofrecer algunas variables tomadas en cuenta en este trabajo, a partir de la revisión de seis números (dos volúmenes) publicados entre 1955-1956 de la revista llamada Suelo Tico perteneciente al Ministerio de Agricultura e Industria de Costa Rica. Esta se consituye en 
un insumo para analizar las pistas que nos acercan al entendimiento de la dinámica alimentaria según el apogeo del sistema benefactor que buscó mejoras en el sector agrario y pecuario por medio de la intervención estatal a inicios de la segunda mitad del siglo XX. Sobre esto último, también se estudiarán las discusiones sobre los peligros o alertas alrededor del productivismo, la lucha tecnológica contra las plagas, y la reorientación de los sistemas de innovación que sugerían utilizar los expertos en la materia de la época.

Asimismo, por la naturaleza de la fuente primaria -la revista Suelo Tico- y el caso específico que se quiere investigar sobre las preocupaciones de la seguridad alimentaria presente en la revista, se analiza solo un fragmento de un largo proceso de intervención estatal hacia la producción alimentaria tradicional (de subsistencia) y la de usos de exportación para la época. De esta manera se intenta tratar de encontrar algunos rasgos, significados o vínculos que unen ese fragmento del contexto de una época y una sociedad más rural: la de los años cincuenta. Este propósito se guió por el siguiente cuestionamiento: ¿cuáles fueron las preocupaciones y misceláneas que se trataron en la revista Suelo Tico en los años 1955-1956 con respecto a las preocupaciones de una noción de seguridad alimentaria?

Lo que nos revelan los datos a la luz de un contexto

Utilizamos para este estudio, una base de datos con 7 variables a tomar en cuenta por medio de los insumos que la información textual de la revista Suelo Tico nos brindaba, organizadas de la siguiente forma en dos gráficos:

- El primer gráfico (Gráfico 2) posee simplemente una pequeña división, el uso de dos variables contrapuestas que encierren cantidad de artículos relacionados a las actividades productivas tradicionales (de subsistencia) y de exportación en la revista Suelo Tico, entre 1955 y 1956. En el primer plano está constituido por producción pecuaria y botánica, apicultura, frutales, forrajes y pastos, algodonera, cacaotera.; y en el segundo plano, por café y caña de azúcar espáticamente.

- El segundo gráfico (Gráfico 3) corresponde a los artículos que hacen referencia a las preocupaciones y misceláneas que se trataron en la revista Suelo Tico, entre 1955 y 1956. Siendo divididas en los siguientes puntos: referencia a la Tecnología y métodos para propiciar o estimular una producción; referencia a los productos que dan beneficios a la salud; referencia a plagas, peligros y medidas preventivas; referencias al campo financiero; referencia a la riqueza geológica y ambiental. Esto va desde la importancia de los productivos de los terrenos de la América tropical y sus combates con las plagas, hasta los baños químicos antiparasitarios para bovinos o usos de maquinaria para aumentar la producción de los cultivos más industriales.

Finalmente, los resultados fueron los siguientes 
A la luz del contexto en que se desarrollan estas actividades productivas se puede decir lo siguiente:

- Aunque las actividades de producción para exportación son mayoritarias, el valor de las actividades tradicionales no es nada despreciable, esto se debe a un contexto de transición tecnológica para incentivar la calidad de los productos. Lo cual sucede en una sociedad todavía con ideas y realidades de subsistencia, sin dejar de lado los grandes productos de exportación que había estado explotando

fuertemente desde fines del siglo XIX. Es interesante como en el Gráfico 3 se da una referencia mayoritaria al factor tecnológico, el conocimiento del ambiente y las plagas. Esto evidencia nuevamente la preocupación no solo de la revista sino nacional por modernizar enteramente una sociedad todavía con rasgos tradicionales, pues hay que tener en cuenta que la revista pertenece a un ministerio estatal. La poca referencia directa al campo financiero y a los beneficios saludables de consumir un productor puede estar más ligada a la falta de conocimiento de esas materias de quienes escriben la revista que a las posibles preocupaciones nacionales con respecto a esos temas (Bonilla, Ramírez y Barrantes, 2001, p. 141; Samper y Naranjo, 2006, pp. 100-107; Solís, 1981, pp. 5562; Rodríguez, 2004, pp. 135-136)

Gráfico 2. Cantidad de artículos relacionados a las actividades productivas tradicionales y de exportación en la revista Suelo Tica, entre 1955-1956. 
- Cantidad de artículos relacionados a las actividades productivas tradicionales (de subsistencia)

Cantidad de artículos relacionados a las actividades productivas de exportación

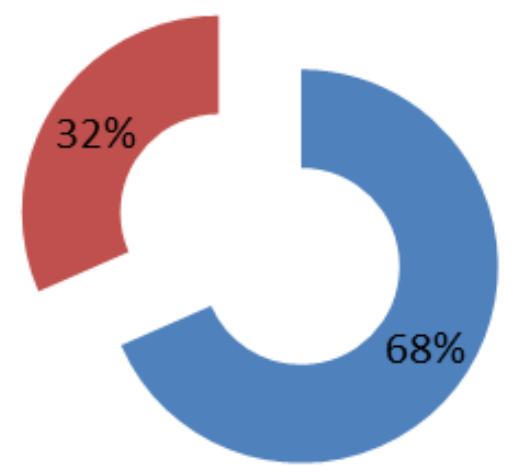

Fuente: Elaboración propia realizado con una base de datos hecha con información del: Ministerio de Agricultura e Industrias, Suelo Tico (revista de divulgación), no. 32-36. San José, 1955-1956.

Gráfico 3. Artículos que hacen referencia a las preocupaciones y misceláneas que se trataron en la revista Suelo Tica, entre 1955 y 1956.

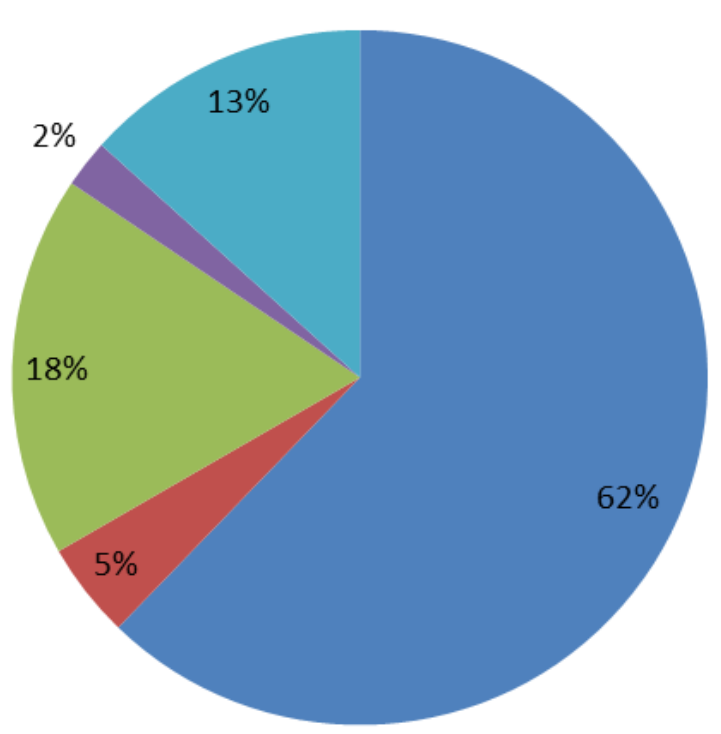

\footnotetext{
- Referencia a la tecnología y métodos para propiciar o estimular una producción

- Referencia a los productos que dan beneficios a la salud
Referencia a plagas, peligros y medidas preventivas

- Referencia al campo financiero

- Referencia a la riqueza geológica y ambiental
} 
Fuente: Elaboración propia realizado con una base de datos hecha con información del: Ministerio de Agricultura e Industrias, Suelo Tico (revista de divulgación), no. 32-36. San José, 1955-1956.

Finalmente, se puede decir que se encontraron insumos no solo gracias a la única fuente primaria (la revista Suelo Tico según se indica en Gráfico 2), sino, también, a la información cuantificada a la luz del contexto brindado por otros estudios. Se logran evidenciar ciertas preocupaciones nacionales por modernizar algunas actividades alimentarias productivas, esto en forma gradual, acumulativa y sin rupturas, en un intento para la incorporación de avances tecnológicos a productos de exportación, lo cual se desarrolló poco a poco pero sin detenerse y congeniando al mismo tiempo con bastantes y variadas actividades de subsistencia que también lograron modernizarse de una u otra manera gracias a las ventajas científicas de la época.

De forma cualitativa podemos observar que desde el año 1957 la importancia en el mejoramiento de los forrajes y su buen uso, a un lado de la importancia de los terrenos de pastoreo y su producción en el contexto Americano tropical según las posibilidades regionales; de ahí que por medio las tierras de pastoreo se dan estudios sobre la adaptación de plantas forrajeras. Aquí el dato más relevante es la extensión agrícola de servicios capacitarios en el desarrollo de las comunidades rurales (Ministerio de Agricultura e Industria, 1957).

En 1958 se habla de la implementación de herbicidas en el café, el combate contra enfermedades no parasitarias del arroz, el cultivo del aguacate y la profilaxis bovina, sin dejar de lado cómo se rescata el papel de la agricultura costarricense en la modernidad del momento (Ministerio de Agricultura e Industria, 1958).

El tema de seguridad o cuidado en los alimentos vuelve aparecer en la revista en 1959 por medio del efecto de las quemas sobre el suelo, el cual aparece como un tema de preocupación nacional. También se sugieren los costos de producción, labores y materiales requeridos en el cultivo de una manzana de cultivo de papa, de donde se rescatan ciertas sugestiones para enfrentarse al problema de las sequías en Costa Rica. Sin embargo, en este año el tema más relacionado con la seguridad alimentaria está presente en la conservación de los suelos como una necesidad mundial (Ministerio de Agricultura e Industria, 1959).

Ya para 1960 y 1961, se ofrece una exposición nacional ganadera con el fin del mejoramiento del ganado lechero en nuestro país, y otros factores que contribuyeron al 
buen éxito de la ganadería en general. Así, se aborda la alimentación foliar de las cosechas, el cultivo de la avena y el control químico de las cebollas, sin dejar de lado nuevamente la situación costarricense durante la época y el desarrollo de la ganadería en San Carlos (Ministerio de Agricultura e Industria, 1960-61).

\section{Origen histórico del derecho alimentario en Costa Rica: la Ley General de la Salud}

El derecho humano a la seguridad alimentaria que hoy en día posee la población costarricense está materializado legalmente en la Política Nacional de Seguridad Alimentaria y Nutricional 2011-2021 (Ministerio de Salud, 2011), la cual fue creada con el fin de:

\footnotetext{
....avanzar en la protección social de la población y asegurar el derecho a la alimentación y nutrición adecuada. Es el instrumento para lograr un sistema nacional de seguridad alimentaria y nutricional que permite guiar y orientar el quehacer institucional y la consecuente asignación de recursos, la integración y la cohesión de los diferentes actores sociales. En ella se establecen cinco ámbitos de acción: disponibilidad, acceso, consumo, utilización biológica y fortalecimiento interinstitucional. (Organización dela Naciones Unidas para la Alimentación y la Agricultura, 2011).
}

Este plan tiene un origen histórico más general, el cual está relacionado con el campo de la salud pública. Nos referimos a algo más antiguo, que deviene de cuando el Ministerio de Salud por medio de la Secretaría de la Política Nacional de Alimentación y Nutrición (SEPAN), un ente adscrito al Despacho del Ministro de Salud, que promovió el cumplimiento de la estrategia COSAN, la cual fue la primera etapa de formulación de la Política Nacional de Alimentación y Nutrición para el período 2006-2010 (FAO, 2016, 32). Asímismo, hay un hito legal más lejano en el tiempo, nos referimos a la Ley General de la Salud de 1973 (Asamblea Legislativa de la República de Costa Rica, 1973), o sea, la ley 5395, fechada del 30 de noviembre de 1973 y que sigue aún en vigencia con algunas reformas el 15 de junio de 1980, el 7 de octubre del 2004 y el 04 de marzo del 2014. Cabe señalar que hubo un veto de varias reformas a ley en 1978.

Una legislación específica en el tema de seguridad alimentaria y nutricional que acompañe el plan es de suma importancia pero es inexistente en Costa Rica, aunque desde los cimientos del primer intento se procura de alguna forma luchar con los problemas alimentarios y nutricionales que aquejaban o aquejan a la sociedad en que vivimos, en forma de órdenes y dictámenes que existen en la Ley General de la Salud de 1973. Así, se trata de mostrar las posibilidades legales por mejorar nuestro derecho alimentario para no partir desde cero, el cual tenemos vigente en Costa Rica desde los años setentas hasta el presente. Al respec tohay artículos relacionados a la seguridad alimentaria y nutricional que deben verse como un intento de permitir el desarrollo social 
y económico de manera sostenida en términos de salud poblacional y sus cuidados, además de la calidad de vida futura para los habitantes.

Asimismo, se evidencia que la legislación costarricense no tiene una Ley de Seguridad Alimentaria y Nutricional 5 como tal, pero que desde los años setentas en materia legal se ha logrado proteger de cierta forma alcances del derecho a la seguridad alimentaria y nutricional de la población, por medio de otros dictámenes presentes en los artículos de la Ley General de la Salud. Esto sucede por lo menos en términos de preocupaciones de consumo de alimentos, donde hay una visión de la seguridad alimentaria que se dedica a gestionar la inocuidad alimentaria para que las condiciones y prácticas se dediquen a preservar la calidad de los alimentos según la contaminación o enfermedades transmitidas por el consumo.

La ley vigente analizada tiene origen en los años setentas, pero para crear un cambio estructural de las futuras políticas que el Estado de Costa Rica, se debería de implementar con el objeto de solucionar los problemas alimentarios que afectan a la sociedad costarricense, más allá del control en los alimentos consumidos, con el fin de mirar hacia otros factores, como por ejemplo, el agrario y la necesidad de una reforma en ese campo que puedan disminuir la pobreza y el desempleo; y de manera informativa esto lleve a disminuir y erradicar la mala alimentación de los pobladores, y hasta ofrezca seguridad alimentaria al país.

Esta Ley General de la Salud tenía como disposiciones generales del derecho a la salud en su primer artículo que "la salud de la población es un bien de interés público tutelado por el Estado"; y luego en el segundo artículo estipulaba que "es función esencial del Estado velar por la salud de la población" (Asamblea Legislativa de la República de Costa Rica, 1973), correspondiendo esto al Poder Ejecutivo por medio del Ministerio de Salubridad Pública. La importancia de los dos primeros artículos que son generalidades, corresponde no sólo porque uno complementa al otro, sino que por primera vez en la historia de Costa Rica se empieza a definir legalmente cómo la política nacional debe estar involucrada en el tema del cuidado poblacional en la salud, formación, planificación y coordinación de todas las actividades tanto públicas como privadas. También, podemos rescatar como fundamento legal de las acciones estratégicas del Ministerio de Salud para el fortalecimiento de la seguridad alimentaria y

nutricional las que se presentan en la Ley General de Salud en su artículo 196, donde se nos habla de un derecho a una nutrición adecuada y la ingestión de alimentos de buena calidad y en condiciones sanitarias, las cuales son esenciales para la salud (Castillo, 2011).

Ahora bien, los siguientes artículos de la ley desde el número 3 hasta el 394, son más específicos entorno a los derechos y deberes ciudadanos en la salud, además de

\footnotetext{
5 Véase la existencia de dicha ley para el caso de Guatemala en Herrera (2011).
} 
complementarios, vigencias, procedimientos e infracciones, cuestión que sólo vamos a rescatar cuando hayan artículos que tengan relación con alguna noción de salud alimentaria o nutricional.

Por ejemplo, en el artículo 12 se estipula que "toda madre gestante tiene derecho a los servicios de información materno-infantil, al control médico durante su embarazo; a la atención médica del parto y a recibir alimentos para completar su dieta, o la del niño, durante el período de lactancia" (Castillo, 2011, s.p.). Este derecho como hemos visto ha sido raíz de toda una lucha tanto poblacional como institucional pública por el que se ha buscado regir su cumplimiento en defensa de un marco legal. Asimismo, en el artículo 14 se hace responsables a los padres o tutor del menor que este en controles médicos según el uso adecuado de los alimentos que reciban como suplementos nutritivos de su dieta. Además, hay toda una reglamentación sobre el uso, modificaciones o venta ilegal de alimentos con uso medicamente adulterado, terapéutico o en complemento de dietas según los artículos 15, 21, 104. Por ejemplo, en la parte b. del artículo 203 se nos habla de que una extracción parcial o total de los componentes de un alimento está haciéndole perder o disminuir su valor nutritivo, viéndose como un alimento adulterado.

Empero, algo similar sucede con otros artículos, pues se nos habla de los alimentos, desde los deberes de las personas o empresa que operan en materia de alimentos y de las restricciones de tales actividades, ya sea de servicios, trasportación o productivas, además de definiciones sobre que se va a considerar como un alimento enriquecido, artificial, alterado o aditivo. Por ejemplo, en el artículo 196, se habla de la nutrición adecuada y la ingestión de alimentos de buena calidad en condiciones sanitarias, así las personas naturales y jurídicas que se ocupen en estas actividades en el consumo de la población están reguladas por órdenes especiales que la respectiva autoridad de salud del caso autorice. También sobre quienes deben ejercer las profesiones de la salud.

Pareciera que la ley está hecha para tratar asuntos más relacionados con lo empresarial, sobre el manejo adecuado de alimentos de consumo nacional o importaciones y exportaciones, que con respecto a las funciones de instituciones públicas en la salud poblacional. No obstante, entorno a la noción de nutrición sí puede ser considerada partidaria de un derecho sobre la seguridad alimentaria nacional. Pues, en el artículo 16 se expresa que "todo escolar deberá someterse a los exámenes médicos y dentales preventivos y participar en los programas de educación sobre salud y en nutrición complementaria que deberán ofrecer los establecimientos educacionales públicos y privados. Así, en el número 69 se consideran que "son establecimientos de atención médica, para los efectos legales y reglamentarios, aquellos que realicen actividades de promoción de la salud..." (Castillo, 2011, s.p.), tomándose en cuenta los aportes a la sociedad dados por las clínicas o particulares profesionales que traten el tema de la recuperación nutricional. 
La preocupación del artículo 198 por entender la función de un alimento enriquecido todo como todo aquel que "...se le han adicionado sustancias en las cantidades recomendadas por los reglamentos a las normas nutricionales con el objeto de reforzar su valor nutritivo" (Castillo, 2011, s.p.) es muy interesante, pues revela una sociedad más entendida o especializada en la materia tecnológica ya para los años setentas, posiblemente debido a una historia de proyectos pioneros de enriquecimiento alimentario desde inicios del siglo XX.

Podríamos afirmar que en la materia de dictámenes dados por el Ministerio de Salud está presente en el artículo 226, donde no sólo se sugiere sino que se obliga a que "todo productor o fabricante de alimentos deberá cumplir con las disposiciones que el Ministerio decrete ordenando el enriquecimiento o equiparación de determinados alimentos a fin de suplir la ausencia o insuficiencia de alimentos nutrientes en la alimentación habitual de la población" (Castillo, 2011, s.p.). Apartado que está muy en consonancia con una política de seguridad alimentaria para contrarrestar cualquier deficiencia en la población costarricense por medio de controlar legalmente los insumos nutritivos de lo que se consume en el país, obligada y directamente. Otra sección tan relevante

y complementaria con lo pasado es el artículo 345, donde se comparte que "sin perjuicio de las demás atribuciones inherentes a su cargo, corresponde especialmente al Ministro en representación del Poder Ejecutivo" para llegar a "determinar de común acuerdo con los organismos correspondientes la política de nutrición de la población y las medidas necesarias para suplementar la dieta cuando sea procedente" (Castillo, 2011, s.p.). Esto no deja duda sobre el poder que se le otorga desde los años setentas hasta el presenta a las instancias gobernativas para organizar y dirigir políticas de nutrición en cualquier ámbito que implique la salud alimentaria y nutricional de los ciudadanos en forma de política nacional, donde todos los agentes de la sociedad tendrían que colaborar sí están involucrados en el ámbito de la salud que toca la ley.

Finalmente, es importante rescatar que el artículo número 393 nos revela como "se mantienen en vigencia los reglamentos y decretos dictados al amparo del Código Sanitario y la legislación anterior (Ley Orgánica del Ministerio de Salubridad Pública), en tanto no se opongan a la presente ley" (Castillo, 2011, s.p.). Así bien, es observable que varios fragmentos de esta ley desde los años setentas en materia del aseguramiento de los alimentos fue y sigue siendo, un asunto de importancia estratégica para el desarrollo sostenible del país en razón de sus efectos e implicaciones sobre la salud pública, donde la seguridad alimentaria, la sanidad pública y el acceso a mercados alimentarios se conjugan entre sí para ofrecer a la población costarricense una noción de seguridad alimentaria y nutricional. Esta aseveración es evidenciable como dato curioso cuando para el año 2010 en el país se implementa una Política Nacional de Inocuidad de los 
Alimentos, rescatándose el uso de las facultades que se confieren en el campo de la inocuidad alimentaria en la Ley General de Salud en los artículos 1, 2, 213, 238, 342, 346 y 349; además de su complemento con los artículos 1, 2, 4, 49, 196, 199, 200, 201, 202, 203, 204, 213, 214, 216, 217, 218, 223, 225 y 236 de la Ley Orgánica del Ministerio de Salud (No 5412) del 08 de noviembre de $1973 .{ }^{6}$ Por ende, sí hay una noción bastante constituida en el derecho alimentario como una rama del derecho sanitario que regula la industria alimentaria es apreciable, pues se surgiera desde los años setentas una defensa contra las adulteraciones y fraudes alimentarios, pues el área de acción legal va desde la producción hasta el consumo de los alimentos.

\section{Conclusión}

El propósito de este trabajo fue plantearse una aproximación del pasado de la Seguridad Alimentaria y Nutricional cuando aún el concepto no existía. Sin embargo, se comprobó por diferentes ámbitos que los intentos para mejorar el estado nutricional de la población fue un hecho, y que el Estado y sus entidades, Sociedades Privadas y Organismos Internacionales plantearon acciones que de alguna manera intervinieron directamente, como los programas de Nutrición Aplicada a menores de edad, con las raciones de alimentos provistos por el PMA y el SNAA. También la reactivación de la producción de bienes de primera necesidad como el STICA o el INCAP. Respondiendo a la pregunta si la Seguridad Alimentaria tuvo realmente un desarrollo significativo, la respuesta es sí, de manera cualitativa y cuantitativa. De manera cualitativa con las aplicaciones de nutrientes y minerales sobre diferentes alimentos, una yodización de la sal, leche enriquecida con minerales o la flourización de las fuentes de agua. También se puede ver la con la aplicación de la tecnología tal como la revista Suelo Tico proponía. Recordemos que en estos dos decenios (los cincuentas y sesentas) no contemplaban un legislación vigente, lo que sucederá después de los años setenta.

De manera cuantitativa se puede contrastar con lo estático que fueron los números de niños por meses intervenidos con leche por mes en las memorias de la Sociedad de la Gota de Leche. Los números de centros que fueron creándose en el periodo de estudio, que comenzaron en 1951, y ya para 1968 se contaban con 48 en diferentes partes del territorio nacional, contando el ambiente rural y urbano. Igualmente es importante mencionar la cantidad de colegios y por ende población estudiantil intervenida por los Programas Educación Nutricional y Huertos que sobrepasaron las diez mil personas en cada uno de las dos etapas.

Ahora bien, a pesar de que hubo numerosas iniciativas, cabe recordar que para el año 1966 aún estaban sin atenderse una significativa parte de la población aun padecía de

\footnotetext{
${ }^{6}$ Por cuestiones de que dicho planeamiento de los ministerios estatales se nos sale de nuestro período de estudio, únicamente estos artículos serán referenciados, véase en: La Gaceta, n. ${ }^{\circ} 84$, del 03 de mayo de 2010. Costa Rica. Asamblea Legislativa. Disponible en: http://www.imprentanacional.go.cr/pub/2010/05/03/COMP_03_05_2010.html
} 
Revista Herencia, Vol. 32 (1), enero-junio, 2019.

déficit ( $57 \%$ de los niños padecían casos de desnutrición crónica). Eso quizás revela una gran limitación en el alcance del trabajo, pues no se pudo corroborar que a raíz del numero de iniciativas, cuanto se pudo reducir años anteriores, pues los datos poblacionales aun no se han encontrado. 


\section{BIBLIOGRAFÍA}

\section{Fuentes primarias}

Asamblea Legislativa de la República de Costa Rica. (1973). Ley General de Salud. Sistema Costarricense de Información Jurídica (SCIJ) núm. 5395 del 30 de octubre de 1973.

CR-AN-AH. Fondo MRREE. Signatura 8822.

CR-AN-AH. Fondo MREE. Signatura 8527.

CR-AN-AH. Fondo MRREE. Signatura 16397.

CR-AN-AH. Fondo Salud. Signatura 212.

CR-AN-AH. Fondo SGLECHE. Signatura oooog1.

CR-AN-AH. Fondo Ministerio de Educación Pública. Signatura 5056.

CR-AN-CR. Fondo SGLECHE. Signatura ooooo8.

Ministerio de Agricultura e Industria. (1943). Memoria del Ministerio de Agricultura e Industrias. San José: Imprenta Nacional.

Ministerio de Agricultura e Industria. (1948). Suelo Tico (Revista de divulgación), Vol. $1, N^{0} .5$.

Ministerio de Agricultura e Industria. (1951). Memoria del Ministerio de Agricultura e Industrias. San José: Imprenta Nacional.

Ministerio de Agricultura e Industria. Suelo Tico (Revista de divulgación), 1955-1961. Números del 32 al 47.

\section{Fuentes Secundarias}

Asamblea Legislativa. (2010). La Gaceta N. ${ }^{\circ} 84$, del 03 de mayo de 2010. Costa Rica: Imprenta Nacional. Disponible en: http://www.imprentanacional.go.cr/pub/2010/05/03/COMP_03_05_2010.html 
Bonilla, H.; Ramírez, O. \& Barrantes, E. (2001). Costa Rica: La disyuntiva agrícola en el período 1905-1925: cultivos de exportación y cultivos de subsistencia. Anuario de Estudios Centroamericanos 27 (2).

Castillo, S. (2011). Acciones estratégicas del Ministerio de Salud para el fortalecimiento de la Seguridad Alimentaria y nutricional. Viceministerio de Salud. Presentación del Ministerio de Salud de Costa Rica.

Clay, E. (2002). Food Security: Concepts and Measurement. Paper for FAO Expert consultation on Trade and Food Security: Conceptualising the Linkage. Roma: FAO.

FAO (2016). Estado de la Segudidad Alimentaria y Nutricional en Costa Rica, Hacia la elaboración de una estrategia de asistencia técnica de la FAO en apoyo a la implementación de la iniciativa América Latina y el Caribe sin hambre. Ciudad de Guatemala: FAO.

FAO-FIDA-PMA. (2014). El estado de la inseguridad alimentaria en el mundo. Roma: FAO.

Herrera, E. (2011). Falta de cumplimiento de la Ley del Sistema de Seguridad Alimentaria y Nutricional por el Estado de Guatemala. Tesis licenciatura, Universidad de San Carlos de Guatemala. Disponible en: http://biblioteca.usac.edu.gt/tesis/o4/04_9271.pdf

León, J. (s.a.). Las políticas económicas en Costa Rica 1890-1950: La primera mitad del siglo XX. Disponible en: http://www.iice.ucr.ac.cr/PoliticaEconomica1890-1950.pdf

Ministerio de Salud de Costa Rica. (2001). Política Nacional de Seguridad Alimentaria y Nutricional, 2011-2021. San José: Ministerio de Salud.

Ministerio de Salud. (2011). Política Nacional de Seguridad Alimentaria y Nutricional 20112021, $1^{\text {a }}$ edición. San José: Ministerio de Salud.

Muñoz, C. \& Scrimshaw, N. (eds). (1996). La transición de la Nutrición y la salud de Costa Rica Democrática. Vargas, W. Desarrollo y Características de los Servicios de Salud y Nutrición para las Comunidades Urbanas y Rurales de Costa Rica. Guatemala: INCAP.

Organización de las Naciones Unidas para la Alimentación y la Agricultura. (2016). Información General: Política Nacional de Seguridad Alimentaria y Nutricional, 2011-2021. Disponible en: https://www.ministeriodesalud.go.cr/index.php/biblioteca-dearchivos/sobre-el-ministerio/politcas-y-planes-en-salud/politicas-en-salud/1106-

politica-nacional-de-seguridad-alimentaria-y-nutricional-2011-2021/file. Accesado 30 octubre 2016

Rodríguez, E. (2004). Costa Rica en el siglo XX. San José: Editorial de la Universidad Estatal a Distancia. 
Samper, M. \& Naranjo, C. (2006). La innovación tecnológica de la agricultura costarricense, 1880-1920. Revista de Historia, 53-54, Escuela de Historia, UNA/Centro de Investigaciones Históricas de América Central, UCR.

Solís, M. (1981). La agroindustria capitalista en el período 1900-1930. Revista de Ciencias Sociales, 21-22, 55-62.

Viales, R. (ed.). (2005). Pobreza e Historia en Costa Rica: Determinantes estructurales y representación sociales del siglo XVII a 1950. Viales, R. El régimen liberal de bienestar y la institucionalización de la pobreza. San José: CIHAC. 